\title{
Analysis of the Dynamic Behavior of the Inner Hair Cell Stereocilia by the Finite Element Method*
}

\author{
Toshihiro MATSUI**, Chihiro NAKAJIMA**, Yuichi YAMAMOTO**, Masayoshi ANDOH**, \\ Koji IIDA $^{* *}$, Michio MURAKOSHI ${ }^{* *}$, Shun KUMANO ${ }^{* *}$ and Hiroshi WADA**
}

The motion of the inner hair cell (IHC) stereocilia, which results in tension in the tip links connected to mechanically gated ion channels, mediates the auditory transduction process. However, it is difficult to directly observe the motion of the stereocilia because of their minute dimensions and complex structure. In this study, to investigate such motion, a finite element method model of the tall, middle and short IHC stereocilia, including the tip and lateral links extending between the stereocilia, was constructed. By applying an analytically estimated fluid force caused by a stimulus of $60 \mathrm{~dB} \mathrm{SPL}$ at $500 \mathrm{~Hz}$ to the model, the dynamic behavior of the stereocilia was analyzed. Numerical results showed that the stereocilia moved in phase and that the maximum tensions of $2.5 \mathrm{fN}$ and $2.1 \mathrm{fN}$ occurred in the tip link connecting the tall and middle stereocilia and in the tip link connecting the middle and short stereocilia, respectively. By contrast, under the condition in which the lateral links were removed, maximum tension in the former increased to $11.6 \mathrm{fN}$, while that in the latter only increased to $2.3 \mathrm{fN}$. It was therefore suggested that the lateral links protect the MET channels located at taller stereocilia against large stimuli and subject the channels located in the same IHC to forces of similar size.

Key Words: Acoustic, Biomechanics, Finite Element Method, Sound, Cochlea, Hair Cell, Stereocilia

\section{Introduction}

The mammalian auditory system is divided into the external, middle and inner ears. Figure 1 shows a schematic of the auditory system of the guinea pig and its relative position within the head. The external ear receives sound waves and transmits them to the middle ear through the external auditory meatus. In the middle ear, the sound waves vibrate the tympanic membrane, and the resulting vibrations are then mechanically transmitted via the ossicles to the cochlea located in the inner ear. The mammalian cochlea consists of a snail-shell-shaped duct filled with lymph fluid as shown in Fig. 2. Sound waves, which reach the cochlea, are transmitted through the cochlear fluid and vibrate the basilar membrane (BM). The organ of Corti (OC), which sits on the BM, transforms the vibration of the BM to electrical signals. As a result, we perceive sound.

* Received 11th July, 2005 (No. 05-4091)

** Department of Bioengineering and Robotics, Tohoku University, 6-6-01 Aoba-yama, Sendai 980-8579, Japan. E-mail:wada@cc.mech.tohoku.ac.jp
Figure 3 shows a cross section of the OC. The OC contains an inner hair cell (IHC) which operates as a sensory receptor of the auditory system. When the OC undergoes vibration, shearing motion of the tectorial membrane

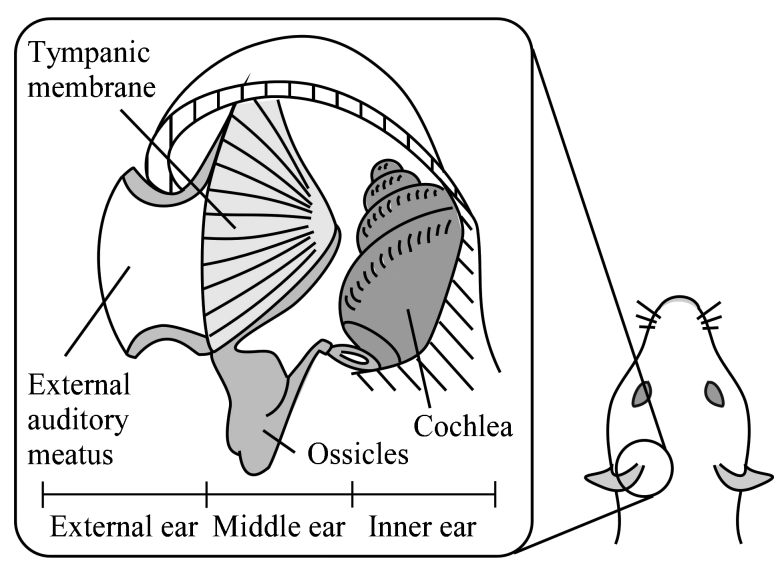

Fig. 1 Schematic of the auditory system of the guinea pig. The external auditory meatus conducts sound waves to the tympanic membrane. The vibration of this membrane is mechanically transmitted via the ossicles to the cochlea. 


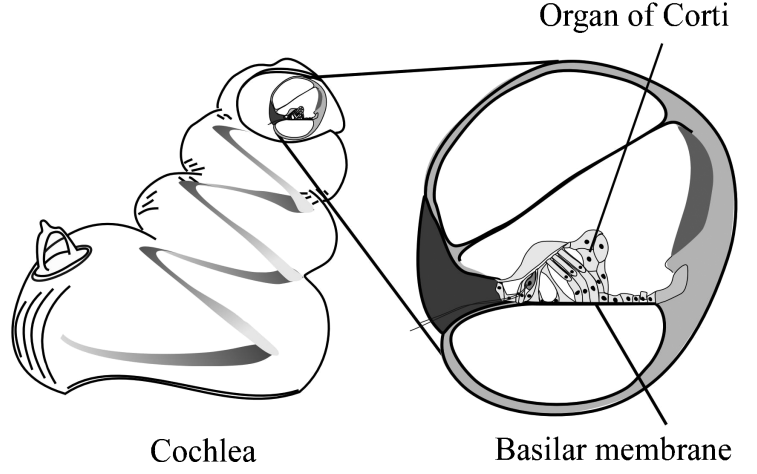

Fig. 2 Schematic of the cross section of the guinea pig cochlea. The cochlea consists of a fluid-filled duct that is coiled like a snail shell. The organ of Corti (OC) sits on the basilar membrane (BM).

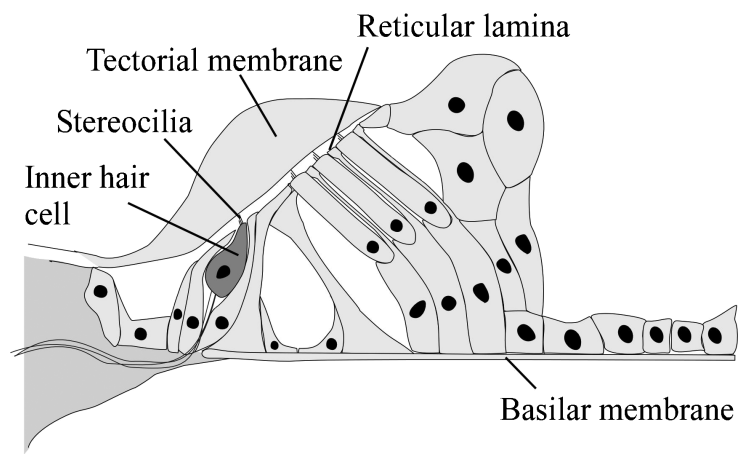

(a)

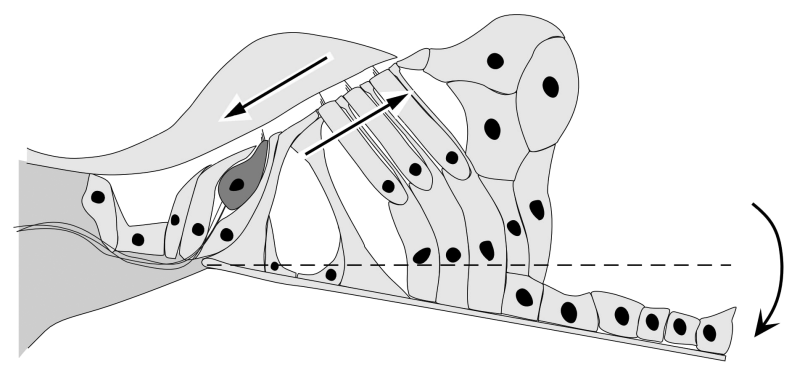

(b)

Fig. 3 Schematic of the cross section of the OC. (a) Resting state of the OC. The OC contains a sensory cell, i.e., the inner hair cell (IHC). (b) Bending state of the OC. When the $\mathrm{BM}$ vibrates vertically, the stereocilia are deflected by the shearing motion of the tectorial membrane (TM) against the reticular lamina (RL) through endolymph.

(TM) against the reticular lamina (RL) occurs ${ }^{(1)}$. A bundle of stereocilia located on the apical surface of the IHC is deflected by Couette flow of the endolymph caused by such shearing motion ${ }^{(1)}$. Figure 4 shows a schematic of the IHC. About sixty stereocilia, a few micrometers in height, are arranged in a staircase pattern and interconnected by two types of links, i.e., tip links, which extend between the tip of the shorter stereocilium and the lateral wall of the next taller one, and lateral links, which extend between the shafts of the stereocilia ${ }^{(2)}$. Figure 5 shows the internal structure of the stereocilium. The stereocilium consists of

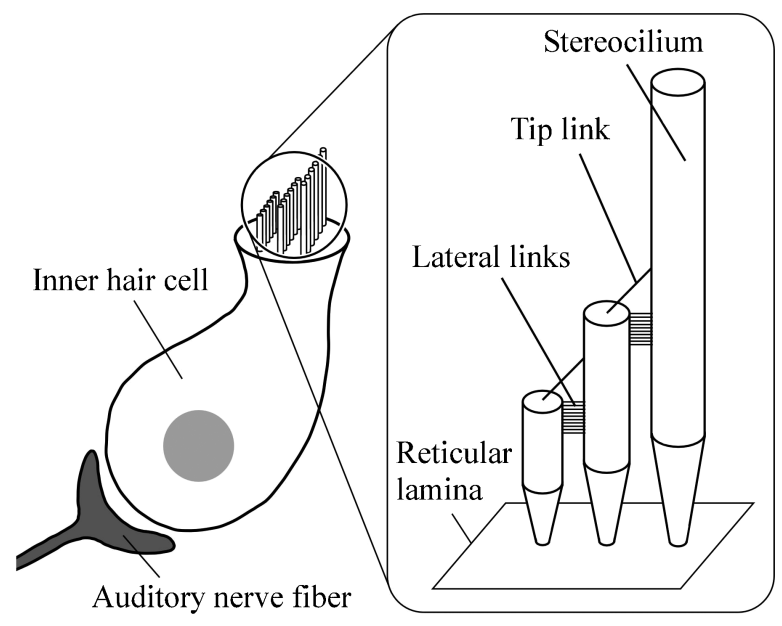

Fig. 4 Schematic of the IHC. The stereocilia are located on the apical surface of the IHC, i.e., the reticular lamina (RL), and are arranged in a staircase pattern. There are two types of links, i.e., tip links, which extend between the tip of the shorter stereocilium and the lateral wall of the next taller one, and lateral links, which extend between the shafts of the stereocilia.

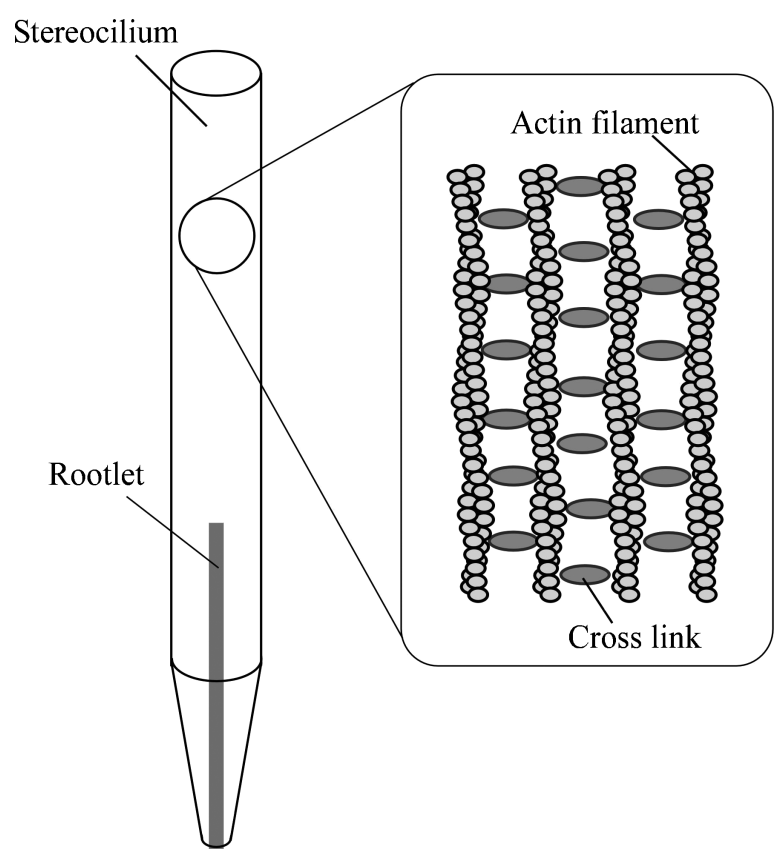

Fig. 5 Internal structure of the stereocilium. The stereocilium consists of cross-linked actin filaments, the so-called intracellular actin cytoskeleton, and the rootlet. The density of the actin filaments which compose the rootlet is larger than that of the intracellular actin cytoskeleton. The rootlet is the core of the stereocilium and makes it stiff.

cross-linked actin filaments ${ }^{(3)-(8)}$, the so-called intracellular actin cytoskeleton, and the rootlet. The rootlet is the core of the stereocilium and is thought to be responsible for its stiffness ${ }^{(9)}$. When the bundle is deflected in the excitatory direction, i.e., toward the tallest stereocilium, the tip link is under tension and the mechanoelectrical trans- 
duction (MET) channel located at the end of the tip link is thought to open ${ }^{(10)}$. Due to this, the membrane potential of the IHC is depolarized and action potentials are then produced in the auditory nerve fibers ${ }^{(11)}$.

To clarify the transduction process of the auditory system, investigation of the motion of the stereocilia, which results in the tension in the tip link, is necessary. However, it is difficult to obtain such motion from experiments because the stereocilia are a few micrometers in height and they interact with one another due to the links ${ }^{(2)}$. Therefore, numerical analyses, which are useful in the investigation of such motion, have been done to date ${ }^{(12)-(14)}$. Zetes and Steele ${ }^{(12)}$ employed the linear, multi-degree of freedom equation of motion for the fluidstructure interaction of the stereocilia and obtained the frequency responses of the displacement of the stereocilia and the tension in the tip links. They showed that viscous force caused by endolymph limited the motion of the stereocilia, resulting in the reduction of the tension in the tip links in a fashion analogous to that of a low pass filter. However, the connection of the lateral link between the stereocilia was not considered. In addition, although all stereocilia are subject to the fluid force caused by the flow of endolymph in vivo, force was applied to only the tip of the tallest stereocilium in their analysis. Duncan and Grant ${ }^{(13)}$ presented a finite element method (FEM) model of the IHC stereocilia and analyzed the deflection of the stereocilia by applying force to the tip of the tallest stereocilium. The relationship between the stiffness of the stereocilium and its Young's modulus was elucidated in their analysis. Cotton and Grant ${ }^{(14)}$ showed the reduction of the stiffness of the stereocilia caused by the buckling of the links by using a three-dimensional FEM model of the vestibular hair cell bundle. However, the dynamic behavior of the bundle was not examined by Duncan and Grant $^{(13)}$ nor by Cotton and Grant ${ }^{(14)}$. Furthermore, in the above three analyses, as the stereocilium was assumed to be homogeneous, the contribution of Young's modulus of the rootlet to the stiffness of the stereocilia was not considered.

In this study, a three-dimensional FEM model of the IHC stereocilia of the guinea pig including the rootlet and the lateral link was constructed based on measurement data reported in the literature ${ }^{(2)-(6),(15)-(21)}$. The unknown Young's modulus was estimated by comparing the numerically obtained stiffness of the stereocilia with that experimentally obtained in previous study ${ }^{(16)}$. Then, by applying the analytically estimated fluid force exerted on the stereocilia caused by the flow of endolymph to the constructed model, the dynamic behavior of the stereocilia was analyzed.

\section{Modeling of Stereocilia}

\subsection{Geometry}

The geometry of the FEM model of the IHC stereocilia located $17 \mathrm{~mm}$ from the basal end in a guinea pig cochlea shown in Fig. 6 (a) was determined from measurement data reported in the literature ${ }^{(2),(15)-(17),(20),(21)}$. The characteristic frequency $(\mathrm{CF}$, the most effective stimulus frequency) at this location is approximately $500 \mathrm{~Hz}^{(22)}$. The numbers of hexahedral elements assigned to the models of tall, middle and short stereocilia were 888, 456 and 336, respectively. As shown in Fig. 6(b), a cylindrical component of the model consists of twelve elements. In the components in which the rootlet exists, four inner elements and eight outer elements represent the rootlet and the intracellular actin cytoskeleton, respectively. On the other hand, in the components in which the rootlet does not exist, all twelve elements represent the intracellular actin cytoskeleton. The boundary condition at the bottom of the model was fixed.

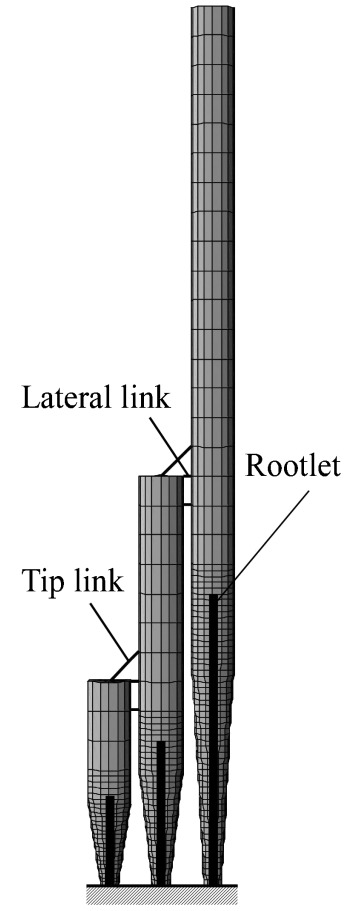

(a)

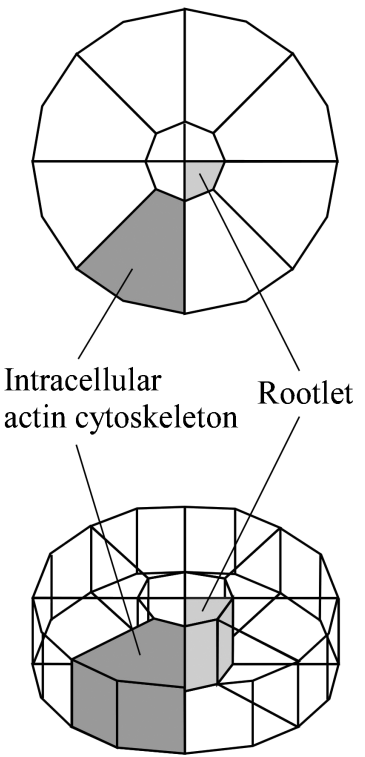

(b)
Fig. 6 FEM model of the stereocilia. (a) Side view of the model. The numbers of hexahedral elements assigned to the models of tall, middle and short stereocilia are 888, 456 and 336, respectively. The boundary condition at the bottom of the model is fixed. (b) Cross-sectional and perspective views of the model. The cylindrical component of the model is composed of twelve elements. In the components in which the rootlet does not exist, all twelve elements represent the intracellular actin cytoskeleton. On the other hand, in the components in which the rootlet exists, four inner elements represent the rootlet. 
Table 1 Geometric parameters assigned to the model

\begin{tabular}{lccc}
\hline \hline & Tall $(\mu \mathrm{m})$ & Middle $(\mu \mathrm{m})$ & Short $(\mu \mathrm{m})$ \\
\hline Stereocilium height & 6.0 & 2.8 & 1.4 \\
Stereocilium diameter & 0.3 & 0.3 & 0.3 \\
Stereocilium base diameter & 0.12 & 0.12 & 0.12 \\
Stereocilium taper height & 1.6 & 0.8 & 0.6 \\
Rootlet height & 2.0 & 1.0 & 0.6 \\
Rootlet diameter & 0.05 & 0.05 & 0.05 \\
\hline \hline & \multicolumn{5}{|}{$283 \mathrm{~nm}$} \\
Tip link length & \multicolumn{5}{|}{$50 \mathrm{~nm}$} \\
Lateral link length &
\end{tabular}

The tip and lateral links were modeled as spring elements formed by two nodes. The tip link was constructed between the center of the tip of the shorter stereocilium and the lateral wall of the next taller one at an angle of 45 degrees, and its length was $283 \mathrm{~nm}^{(15),(20)}$. In this study, the tip link connecting the tall and middle stereocilia and that connecting the middle and short stereocilia were termed the first tip link and the second tip link, respectively.

It is known that a single tip link connects neighboring stereocilia, while ten lateral links connect adjacent stereocilia for a length of about $200 \mathrm{~nm}$ along their shafts ${ }^{(21)}$. However, ten lateral links could not be separately applied to the model because of the location of nodes on the elements. In this study, to represent the connection of the lateral links, two spring elements were constructed horizontally between adjacent stereocilia, and a spring constant, which is five times larger than that of a single lateral link, was assigned to each spring element. The upper element was located between the tip of the shorter stereocilium and the lateral wall of the next taller one, and the lower element was located $200 \mathrm{~nm}$ below the upper one. The length of each element was determined to be $50 \mathrm{~nm}$ based on the above-cited study ${ }^{(21)}$. The geometric parameters assigned to the FEM model of the IHC stereocilia are shown in Table 1.

\subsection{Mechanical properties}

To analyze the dynamic behavior of the IHC stereocilia by using FEM, values of the density, Poisson's ratio and Young's modulus of the intracellular actin cytoskeleton and the rootlet, which were constructed of hexahedral elements, and values of the spring constant of the tip and lateral links, which were constructed of spring elements, were necessary. The densities of the intracellular actin cytoskeleton and the rootlet were determined to be $1.2 \times 10^{3} \mathrm{~kg} / \mathrm{m}^{3}$, which is the median of the range from that of water to that of protein, i.e., from $1.0 \times 10^{3} \mathrm{~kg} / \mathrm{m}^{3}$ to $1.4 \times 10^{3} \mathrm{~kg} / \mathrm{m}^{3(23)}$. Poisson's ratios of the intracellular actin cytoskeleton and the rootlet were assumed to be 0.4 because they were biomaterials, i.e., nearly incompressible materials ${ }^{(13)}$.

Young's modulus of the intracellular actin cytoskeleton $E_{\mathrm{IAC}}$ was expressed by

$$
E_{\mathrm{IAC}}=\frac{203 E I}{l^{4}},
$$

where $E I$ is the flexural rigidity of actin filament and $l$ is the length of the cross-link ${ }^{(4),(5)}$, i.e., the distance between the actin filaments as shown in Fig. 5. Based on the reported measurement data ${ }^{(3),(4),(18)}, E I$ and $l$ were estimated to be $1.7 \times 10^{-25} \mathrm{Nm}^{2}$ and $25 \mathrm{~nm}$, respectively. From Eq. (1), Young's modulus of the intracellular actin cytoskeleton $E_{\mathrm{IAC}}$ was then determined to be $8.6 \times 10^{7} \mathrm{~Pa}$.

The spring constant of the tip and lateral links $K$ was given by

$$
K=\frac{E A}{L},
$$

where $A, L$ and $E$ are the cross-sectional area, the length and Young's modulus of these links, respectively. In this study, the tip and lateral links were assumed to be a column with a diameter of $8.0 \mathrm{~nm}^{(20)}$, resulting in a crosssectional area $A$ of $50.3 \mathrm{~nm}^{2}$. As mentioned in the previous section, the length of the tip link $L_{\mathrm{TL}}$ and that of the lateral link $L_{\mathrm{LL}}$ were $283 \mathrm{~nm}$ and $50 \mathrm{~nm}$, respectively. Young's modulus of the tip link $E_{\mathrm{TL}}$ was determined to be $3.0 \times 10^{6} \mathrm{~Pa}$ based on the previous study ${ }^{(19)}$. By substituting these parameters into Eq. (2), the spring constant of the tip link was determined to be $5.3 \times 10^{-4} \mathrm{~N} / \mathrm{m}$.

Young's moduli of the rootlet and the lateral link have remained unknown. Thus, these Young's moduli were estimated by using the FEM model of the IHC stereocilia in section 3 .

\subsection{Fluid force exerted on stereocilia caused by flow of endolymph}

The fluid force exerted on the stereocilia caused by the flow of endolymph was analytically estimated. When the BM vibrates vertically, shearing motion of the TM against the RL occurs. Assuming the existence of Couette flow between the TM and RL caused by this shearing motion as shown in Fig. 7 (a), the fluid velocity for Couette flow $V$ is given by

$$
V=\frac{z}{H} V_{\mathrm{TM}}
$$

where $z$ is the axis perpendicular to the RL, $H$ is the distance between the TM and the RL and $V_{\mathrm{TM}}$ is the velocity of the TM relative to the RL. The distance $H$ was determined to be $7.3 \mu \mathrm{m}$ based on reported measurement data $^{(24)}$. To analyze the dynamic behavior of the stereocilia in section $4, V_{\mathrm{TM}}$ caused by stimuli of $60 \mathrm{~dB}$ SPL at $500 \mathrm{~Hz}$ was determined to be $1.3 \times 10^{-5} \mathrm{~m} / \mathrm{s}$ by using a two-dimensional FEM model of the OC of the guinea pig (Nakajima, personal communication), which was reconstructed based on a previously reported OC model ${ }^{(25)}$. 


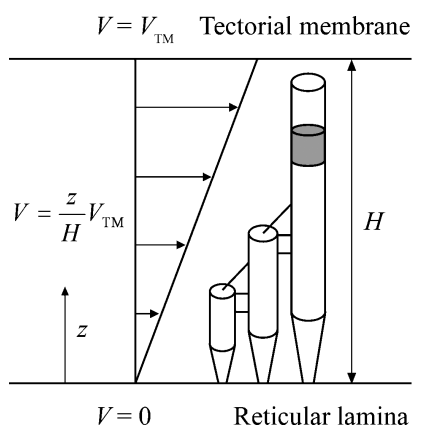

(a)

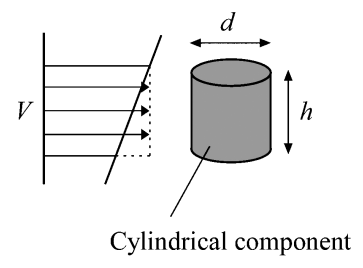

(b)
Fig. 7 Flow of the endolymph around the stereocilia. (a) Schematic of Couette flow between the TM and the RL. The shearing motion of the TM against the RL causes Couette flow between them. (b) Schematic of the flow around a cylindrical component of the stereocilia in the FEM model.

In this study, the force exerted on each cylindrical component of the stereocilia in the FEM model was considered as shown in Fig. 7 (b). Based on the study of Tomotika and Aoi ${ }^{(26)}$, the fluid force $F$ exerted on the cylindrical component was obtained as

$$
F=\frac{4 \pi \mu h}{S} \frac{z}{H} V_{\mathrm{TM}}
$$

where $h$ is the height of the cylindrical component, which is taken to be $200 \mathrm{~nm}$, and $\mu$ is the viscosity coefficient of endolymph, which is assumed to be that of water, i.e., $0.659 \times 10^{-3} \mathrm{~Pa} \cdot \mathrm{s}$. The variable $S$ in Eq. (4) is expressed by

$$
S=\frac{1}{2}-\gamma-\log \frac{R_{\mathrm{e}}}{8},
$$

where $\gamma=0.57721$ is Euler number and $R_{\mathrm{e}}$ is Reynolds number, the latter being defined as

$$
R_{\mathrm{e}}=\frac{\rho d}{\mu} \frac{z}{H} V_{\mathrm{TM}},
$$

where $d$ is the diameter of the cylindrical component, i.e., that of the stereocilium, and $\rho$ is the density of endolymph, which is assumed to be that of water, i.e., $1.0 \times 10^{3} \mathrm{~kg} / \mathrm{m}^{3}$. The diameter $d$ varies with $z$ from the diameter of the base of the stereocilium to that of its shaft, i.e., from $0.12 \mu \mathrm{m}$ to $0.3 \mu \mathrm{m}$ as described in Table 1 . By substituting the fluid force $F$ exerted on each cylindrical component obtained from Eq. (4) into the force term in the equation of motion for the FEM model of the stereocilia, which is described in the following section, the dynamic behavior of the stereocilia was analyzed.

\section{4 Equation of motion of stereocilia}

The equation of motion for the FEM model of the stereocilia is represented by the following matrix differential equation:

$$
[\mathrm{M}] \frac{\partial^{2} \mathbf{u}}{\partial t^{2}}+[\mathrm{C}] \frac{\partial \mathbf{u}}{\partial t}+[\mathrm{K}] \mathbf{u}=\mathbf{f},
$$

where $[\mathrm{M}],[\mathrm{C}]$ and $[\mathrm{K}]$ are the mass, damping and stiffness matrices, respectively, $\mathbf{u}$ is the displacement vector, $\mathbf{f}$ is the force vector and $t$ is the time. The force vector f corresponds to the fluid force exerted on the stereocilia as mentioned in section 2.3. The damping matrix [C] was determined as detailed below.

When stereocilia move through fluid, they are subjected to a drag force. The drag force $F_{\text {drag }}$ experienced by a cylindrical component, which constitutes the FEM model of the stereocilia, is given by

$$
F_{\text {drag }}=\frac{4 \pi \mu l}{S} v,
$$

where $v$ is the velocity of the cylindrical component and $S$ is the same value represented in Eq. (5). Under this condition, Reynolds number $R_{\mathrm{e}}$ is defined as

$$
R_{\mathrm{e}}=\frac{\rho d v}{\mu} \text {. }
$$

Since $F_{\text {drag }}$ is proportional to the velocity of the cylindrical component, this drag force was assumed to be the viscous damping force. The damping coefficient $C_{\text {damp }}$ was therefore determined as follows:

$$
C_{\text {damp }}=\frac{4 \pi \mu l}{S} \text {. }
$$

Equations (5), (9) and (10) lead to the fact that $C_{\text {damp }}$ varies with the velocity $v$. In this study, to make $C_{\text {damp }}$ constant, $v$ was assumed to be the maximum velocity of the cylindrical component which was obtained from Eq. (7) under the condition in which the viscous damping force was not considered. By inserting the damping coefficient $C_{\text {damp }}$ of each cylindrical component obtained from Eq. (10) into the damping matrix [C], the damping force caused by the movement of the stereocilia through fluid was considered.

\section{Estimation of Unknown Young's Moduli}

Young's moduli of the rootlet and the lateral link are unknown. In this study, Young's modulus of the rootlet is assumed to be greater than that of the intracellular actin cytoskeleton, i.e., $8.6 \times 10^{7} \mathrm{~Pa}$, and smaller than that of the actin filament, i.e., $2.6 \times 10^{9} \mathrm{~Pa}^{(18)}$, because Young's modulus depends on the density of actin filaments, and this density in the rootlet is thought to be greater than that of the intracellular actin cytoskeleton ${ }^{(27),(28)}$ and smaller than that of the actin filament itself.

To estimate Young's modulus of the lateral link, the numerically obtained stiffness of the stereocilia, which was defined as the force required to produce a unit deflection of the tall stereocilium, was compared with that experimentally obtained in previous study ${ }^{(16)}$. Figure 8 shows the relationship between the stiffness of the stereocilia and Young's modulus of the lateral link for various Young's moduli of the rootlet. The two horizontal broken lines in Fig. 8 represent the maximum and minimum stiffness of the stereocilia located $18 \mathrm{~mm}$ from the basal end in a guinea pig cochlea experimentally obtained in previous study ${ }^{(16)}$. By comparing the numerically obtained stiffness with that experimentally obtained, the range of 


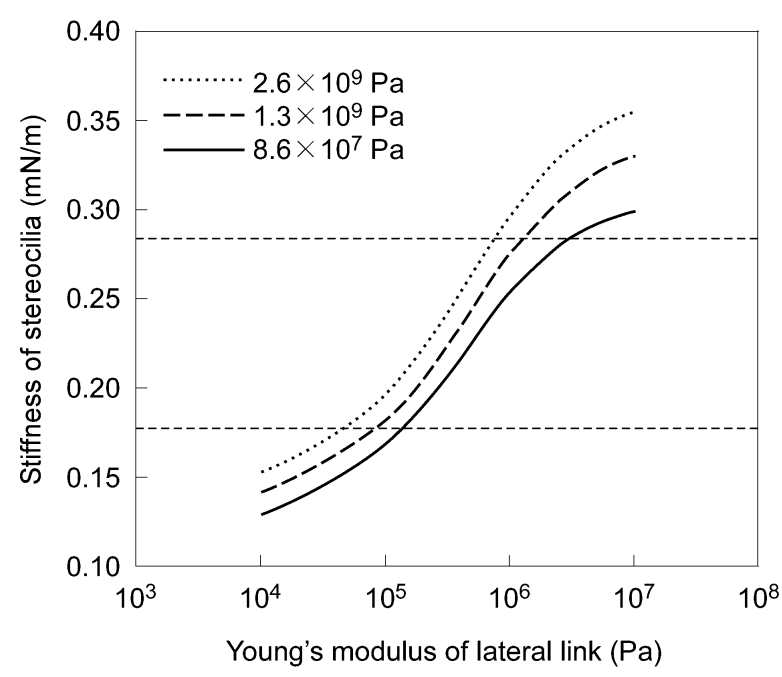

Fig. 8 Relationship between the stiffness of the stereocilia and Young's modulus of the lateral link for various Young's moduli of the rootlet. The notations in the figure represent Young's moduli of the rootlet. The two horizontal broken lines represent the maximum and minimum stiffness of the stereocilia experimentally obtained in a previous study ${ }^{(14)}$.

Table 2 Mechanical properties assigned to the model

\begin{tabular}{ll}
\hline Young's modulus $(\mathrm{Pa})$ & \\
Rootlet & $1.3 \times 10^{9}$ \\
Intracellular actin cytoskeleton & $8.6 \times 10^{7}$ \\
Tip link & $3.0 \times 10^{6}$ \\
Lateral link & $5.0 \times 10^{5}$ \\
Density $\left(\mathrm{kg} / \mathrm{m}^{3}\right)$ & $1.2 \times 10^{3}$ \\
Poisson's ratio & 0.4 \\
\hline
\end{tabular}

Young's modulus of the lateral link was estimated to be from $1.0 \times 10^{5} \mathrm{~Pa}$ to $1.0 \times 10^{6} \mathrm{~Pa}$. Moreover, the stiffness of the stereocilia increased $17 \%$ when Young's modulus of lateral link was maintained at $5.0 \times 10^{5} \mathrm{~Pa}$ and that of the rootlet was increased from $8.6 \times 10^{7} \mathrm{~Pa}$ to $2.6 \times 10^{9} \mathrm{~Pa}$.

For the following analysis, Young's moduli of the rootlet and the lateral link were determined to be $1.3 \times$ $10^{9} \mathrm{~Pa}$ and $5.0 \times 10^{5} \mathrm{~Pa}$, respectively, each of which was the median of the range of its Young's modulus. Under this condition, the spring constant of each spring element which represents the five lateral links was determined to be $2.5 \times 10^{-3} \mathrm{~N} / \mathrm{m}$ from Eq. (2). All mechanical properties assigned to the model are shown in Table 2 .

\section{Analysis of Dynamic Behavior of Stereocilia}

The dynamic behavior of the stereocilia was analyzed under the condition in which the fluid force caused by the stimulus of $60 \mathrm{~dB}$ SPL at $500 \mathrm{~Hz}$ was applied to the model. Figure 9 (a) shows the time course of the angular displacements of the tips of the stereocilia. All three stere-

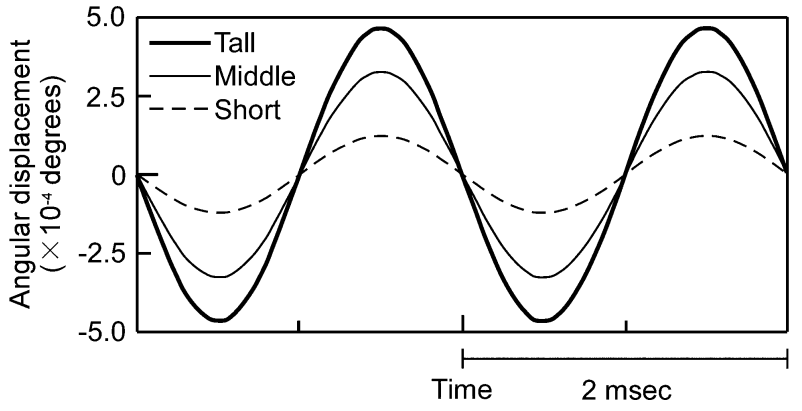

(a)

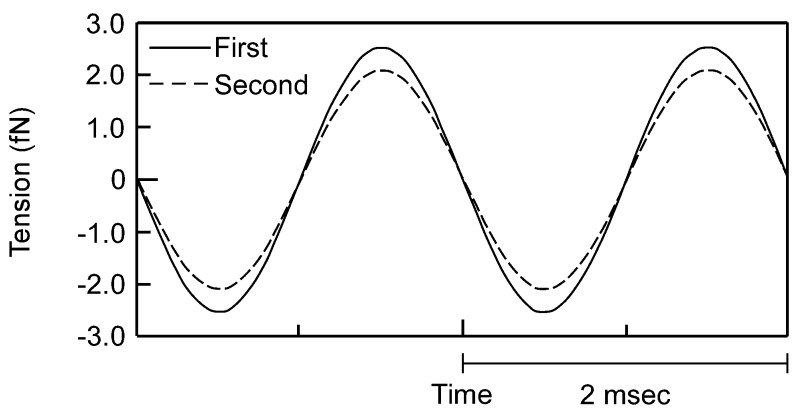

(b)

Fig. 9 Dynamic behavior of the stereocilia under the condition in which the fluid force caused by a stimulus of $60 \mathrm{~dB}$ SPL at $500 \mathrm{~Hz}$ is applied to the model. (a) Time course of the angular displacements of the tips of the stereocilia. Positive displacement of the stereocilium is defined as that in the excitatory direction. (b) Time course of the tension in the tip links.

ocilia moved in phase and showed symmetric displacement in the excitatory direction and the opposite direction. The largest angular displacements of the tall, middle and short stereocilia in both directions were $4.7 \times 10^{-4}$ degrees, $3.3 \times 10^{-4}$ degrees and $1.2 \times 10^{-4}$ degrees, respectively. These angular displacements were equivalent to the horizontal displacements of $48.9 \mathrm{pm}, 15.5 \mathrm{pm}$ and $3.3 \mathrm{pm}$, respectively. Figure 9 (b) shows the time course of the tension in the tip links. The tension in the first tip link connecting the tall and middle stereocilia and that in the second tip link connecting the middle and short stereocilia changed in phase. The maximum tensions of $2.5 \mathrm{fN}$ and $2.1 \mathrm{fN}$ occurred in the first and second tip links, respectively, and the ratio of the tension in the first tip link to that in the second tip link was 1.19.

To investigate the effect of the lateral links on the dynamic behavior of the stereocilia, this behavior was analyzed under the condition in which the lateral links were removed and the same stimulus was applied to the model. The time courses of the angular displacements of the tips of the stereocilia and the tension in the tip links under this condition are shown in Fig. 10. As well as the dynamic behavior under the condition in which the lateral links were included, the three stereocilia moved in phase, and the tension in the first tip link and that in the second tip 


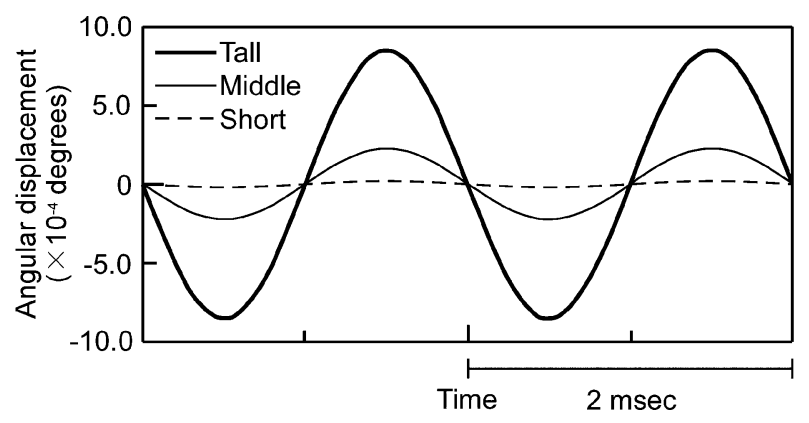

(a)

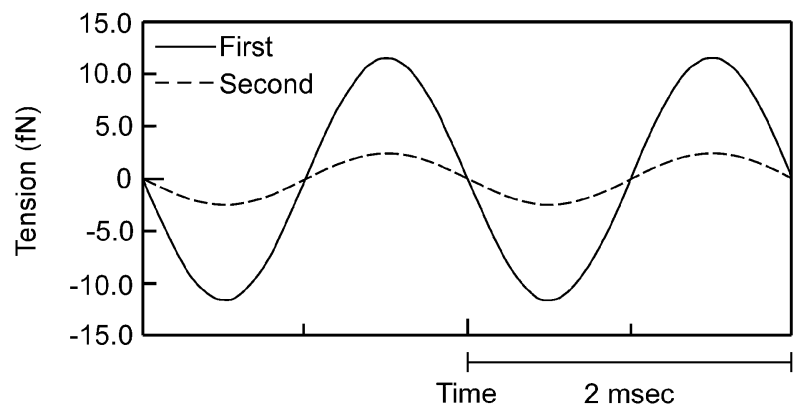

(b)

Fig. 10 Dynamic behavior of the stereocilia when the lateral links were removed. (a) Time course of the angular displacements of the tips of the stereocilia. Positive displacement of the stereocilium is defined as that in the excitatory direction. (b) Time course of the tension in the tip links.

link changed in phase. The largest angular displacements of the tall, middle and short stereocilia in both directions were $8.5 \times 10^{-4}$ degrees, $2.3 \times 10^{-4}$ degrees and $1.8 \times 10^{-5}$ degrees, respectively. The maximum tensions of $11.6 \mathrm{fN}$ and $2.3 \mathrm{fN}$ occurred in the first and second tip links, respectively, and the ratio of the tension in the first tip link to that in the second tip link was 5.04.

\section{Discussion}

Clark and Pickles ${ }^{(29)}$ reported that many IHC stereocilia withstood a 30-min exposure of $115 \mathrm{~dB}$ SPL at $10 \mathrm{kHz}$; however, the stereocilia of the tallest row separated from the shorter stereocilia, although the shorter stereocilia remained interconnected by tip links. In the present analysis, under the condition in which the lateral links were included and a stimulus of $60 \mathrm{~dB} \mathrm{SPL}$ at $500 \mathrm{~Hz}$ was applied to the model, the first tip link was always under a 1.19 times greater stress than the second tip link as shown in Fig. 9(b), the vulnerability of the first tip link thus being greater than that of the second tip link. The numerical result obtained from the present analysis therefore supports their measurement result.

Under the above-mentioned condition, the tension in the first tip link and that in the second tip link changed in phase. Since the tension in the tip link is thought to open the MET channel, this result indicates that all MET chan- nels located in the stereocilia open and close in phase and that a periodic change of the receptor potential of the IHC occurs. Mountain and $\mathrm{Cody}^{(30)}$ revealed that the receptor potential of the IHC showed a sinusoidal pattern under the condition in which the guinea pig cochlea was presented with a stimulus of $60 \mathrm{~dB}$ SPL at $525 \mathrm{~Hz}$. Therefore, the numerical result obtained from the present analysis is in agreement with their measurement result.

The dynamic behavior of the stereocilia shown in our study was different from that shown by Zetes and Steele ${ }^{(12)}$, i.e., the three stereocilia moved in phase in our study as shown in Fig. 9(a), whereas they did not move in phase at a stimulus frequency of $500 \mathrm{~Hz}$ in their study. Our study differed from theirs in three ways. Firstly, our method of applying force to the model was different from theirs. Since fluid forces caused by the flow of endolymph are exerted on all stereocilia in vivo, these forces were applied to the three stereocilia in our study, while a force was applied to only the tip of the tallest stereocilium in theirs. Secondly, the connection of the lateral links was taken into account in our study, while it was ignored in theirs. Thus, the stereocilia in our study were more tightly coupled than those in their study. Finally, Young's modulus assigned to the model was different between the two studies. In our study, based on the measurement data, Young's moduli of the intracellular actin cytoskeleton and the rootlet were determined to be $8.6 \times 10^{7} \mathrm{~Pa}$ and $1.3 \times 10^{9} \mathrm{~Pa}$, respectively. By contrast, in their study, the stereocilium was assumed to be homogeneous, and its Young's modulus was estimated to be $1.0 \times 10^{7} \mathrm{~Pa}$ because of the absence of an experimental value. To identify the factor which is responsible for the in-phase motion of the stereocilia, the state of each of the above-mentioned three aspects of our study was adjusted to that in the study of Zetes and Steele ${ }^{(12)}$, and the dynamic behavior of the stereocilia under each condition was analyzed. The results showed that the stereocilia did not move in phase only under the condition in which a force was applied to only the tip of the tall stereocilium, the lateral links were removed and a value of $1.0 \times 10^{7} \mathrm{~Pa}$ was assigned to the stereocilia, i.e., the condition imposed by Zetes and Steele ${ }^{(12)}$. It is considered that the stimulus frequency of $500 \mathrm{~Hz}$ is close to the resonance frequencies of the three stereocilia, so that the displacements of these stereocilia showed the different phase delays relative to the applied force. Consequently, the stereocilia did not move in phase under the abovementioned condition. By contrast, under the same condition except when values of $8.6 \times 10^{7} \mathrm{~Pa}$ and $1.3 \times 10^{9} \mathrm{~Pa}$ were assigned to the intracellular actin cytoskeleton and the rootlet, the stereocilia moved in phase. Under this condition, the resonance frequencies of the tall, middle and short stereocilium were determined to be $125 \mathrm{kHz}$, $453 \mathrm{kHz}$ and $797 \mathrm{kHz}$, respectively. These resonance frequencies are therefore much higher than the stimulus fre- 
quency of $500 \mathrm{~Hz}$, resulting in the in-phase motion of the stereocilia.

Under the condition in which the stimulus of $60 \mathrm{~dB}$ SPL at $500 \mathrm{~Hz}$ was applied to the model, it was found that the tension in the first tip link increased from $2.5 \mathrm{fN}$ to $11.6 \mathrm{fN}$ when the lateral links were removed, while that in the second tip link only increased from $2.1 \mathrm{fN}$ to $2.3 \mathrm{fN}$, as shown in Figs. 9 (b) and 10 (b). It was also found that the ratio of the tension in the first tip link to that in the second tip link rose from 1.19 to 5.04 under the abovementioned condition. These results imply that the lateral links protect the MET channels located in taller stereocilia against large stimuli caused by intense sound presented to the cochlea and subject the channels located in the same IHC to forces of similar size. Assuming that the forces required to open the MET channels were equal between the channels located in the same IHC, this result implies that receptor currents of similar size flow into the IHC through MET channels.

\section{Conclusions}

Based on measurement data reported in the literature, a three-dimensional FEM model of the IHC stereocilia of the guinea pig was constructed. By applying the analytically estimated fluid force caused by the flow of endolymph to the model, the dynamic behavior of the stereocilia was analyzed. The following findings were obtained.

(1) The range of Young's modulus of the rootlet and that of the lateral link were determined to be from $8.6 \times$ $10^{7} \mathrm{~Pa}$ to $2.6 \times 10^{9} \mathrm{~Pa}$ and from $1.0 \times 10^{5} \mathrm{~Pa}$ to $1.0 \times 10^{6} \mathrm{~Pa}$, respectively. When Young's modulus of the rootlet was varied in this range, the stiffness of the stereocilia changed up to $17 \%$.

(2) When the fluid force caused by the stimulus of $60 \mathrm{~dB}$ SPL at $500 \mathrm{~Hz}$ was applied to the model, the three stereocilia moved in phase. The largest angular displacements of the tall, middle and short stereocilia were $4.7 \times 10^{-4}$ degrees, $3.3 \times 10^{-4}$ degrees and $1.2 \times 10^{-4}$ degrees, respectively. The maximum tensions of $2.5 \mathrm{fN}$ and $2.1 \mathrm{fN}$ occurred in the first and second tip links, respectively, the tension in the former being 1.19 times greater than that in the latter.

( 3 ) When the lateral links were removed, the tension in the first tip link increased significantly, while that in the second tip link hardly changed. As a result, the ratio of the tension in the first tip link to that in the second tip link rose to 5.04. These results imply that the lateral links protect the MET channels located in taller stereocilia against large stimuli and subject the channels located in the same IHC to forces of similar size.

\section{Acknowledgements}

This work was supported by a grant from the Human Frontier Science Program, by a Health and Labor Science Research Grant from the Ministry of Health, Labor and Welfare of Japan, and by Grant-in-Aid for Scientific Research on Priority Areas 15086202 from the Ministry of Education, Culture, Sports, Science and Technology of Japan.

\section{References}

( 1 ) Hu, X., Evans, B.N. and Dallos, P., Direct Visualization of Organ of Corti Kinematics in a Hemicochlea, J. Neurophysiol., Vol.82 (1999), pp.2798-2807.

(2) Ishiyama, E. and Ishiyama, K., Atlas of the Inner Ear Morphology, 2nd ed., (in Japanese), (2002), pp.9-10, Sozo Publishing.

( 3 ) Tilney, L.G. and Tilney, M.S., Functional Organization of the Cytoskeleton, Hear. Res., Vol.22 (1986), pp.5577.

( 4 ) You, L., Cowin, S.C., Schaffler, M.B. and Weinbaum, S., A Model for Strain Amplification in the Actin Cytoskeleton of Osteocytes due to Fluid Drag on Pericellular Matrix, J. Biomech., Vol.34 (2001), pp.13751386.

( 5 ) Han, Y., Cowin, S.C., Schaffler, M.B. and Weinbaum, S., Mechanotransduction and Strain Amplification in Osteocyte Cell Processes, Proc. Natl. Acad. Sci. USA, Vol.101 (2004), pp.16689-16694.

( 6 ) Lin, H.W., Schneider, M.E. and Kachar, B., When Size Matters: The Dynamic Regulation of Stereocilia Lengths, Curr. Opin. Cell Biol., Vol.17 (2005), pp.5561.

( 7 ) DeRosier, D.J., Tilney, L.G. and Egelman, E., Actin in the Inner Ear: The Remarkable Structure of the Stereocilium, Nature, Vol.287 (1980), pp.291-296.

( 8 ) Tilney, L.G., Egelman, E.H., DeRosier, D.J. and Saunders, J.C., Actin Filaments, Stereocilia, and Hair Cells of the Bird Cochlea. II. Packing of Actin Filaments in the Stereocilia and in the Cuticular Plate and What Happens to the Organization When the Stereocilia Are Bent, J. Cell Biol., Vol.96 (1983), pp.822834.

( 9 ) Flock, A., Flock, B. and Murray, E., Studies on the Sensory Hairs of Receptor Cells in the Inner Ear, Acta OtoLaryngol., Vol.83 (1977), pp.85-91.

(10) Pickles, J.O., Comis, S.D. and Osborne, M.P., CrossLinks between Stereocilia in the Guinea Pig Organ of Corti, and Their Possible Relation to Sensory Transduction, Hear. Res., Vol.15 (1984), pp.103-112.

(11) Katz, B. and Miledi, R., A Study of Synaptic Transmission in the Absence of Nerve Impulses, J. Physiol. (London), Vol.192 (1967), pp.407-436.

(12) Zetes, D.E. and Steele, C.R., Fluid-Structure Interaction of the Stereocilia Bundle in Relation to Mechanotransduction, J. Acoust. Soc. Am., Vol.101 (1997), pp.3593-3601.

(13) Duncan, R.K. and Grant, J.W., A Finite-Element Model of Inner Ear Hair Bundle Micromechanics, 
Hear. Res., Vol.104 (1997), pp.15-26.

(14) Cotton, J.R. and Grant, J.W., A Finite Element Method for Mechanical Response of Hair Cell Ciliary Bundles, J. Biomech. Eng., Vol.122 (2000), pp.44-50.

(15) Hackney, C.M. and Furness, D.N., Mechanotransduction in Vertebrate Hair Cells: Structure and Function of the Bundle, Am. J. Physiol., Vol.268 (1995), pp.1-13.

(16) Strelioff, D. and Flock, Å., Stiffness of Sensory-Cell Hair Bundles in the Isolated Guinea Pig Cochlea, Hear. Res., Vol.15 (1984), pp.19-28.

(17) Furness, D.N., Zetes, D.E. and Hackney, C.M., Kinematic Analysis of Shear Displacement as a Means for Operating Mechanotransduction Channels in the Contact Region between Adjacent Stereocilia of Mammalian Cochlear Hair Cells, Proc. R. Soc. Lond. B, Vol.264 (1997), pp.45-51.

(18) Gittes, F., Mickey, B., Nettleton, J. and Howard, J., Flexural Rigidity of Microtubules and Actin Filaments Measured from Thermal Fluctuations in Shape, J. Cell Biol., Vol.120 (1993), pp.923-934.

(19) Howard, J. and Hudspeth, A.J., Compliance of the Hair Bundle Associated with Gating of Mechanoelectrical Transduction Channels in the Bullfrog's Saccular Hair Cell, Neuron, Vol.1 (1988), pp.189-199.

(20) Kachar, B., Parakkal, M., Kurc, M., Zhao, Y. and Gillespie, P.G., High-Resolution Structure of Hair-Cell Tip Links, Proc. Natl. Acad. Sci. USA, Vol.97 (2000), pp.13336-13341.

(21) Tsuprun, V. and Santi, P., Structure of Outer Hair Cell Stereocilia Side and Attachment Links in the Chinchilla Cochlea, J. Histochem. Cytochem., Vol.50 (2002), pp.493-502.

(22) Greenwood, D.D., A Cochlear Frequency-Position Function for Several Speices-29 Years Later, J.
Acoust. Soc. Am., Vol.87 (1990), pp.2592-2605.

(23) Loo, B.W., Jr., Sauerwald, I.M., Hitchcock, A.P. and Rothman, S.S., A New Sample Preparation Method for Biological Soft X-Ray Microscopy: Nitrogen-Based Contrast and Radiation Tolerance Properties of Glycol Methacrylate-Embedded and Sectioned Tissue, J. Microsc., Vol.204 (2001), pp.69-86.

(24) Ulfendahl, M., Flock, A. and Scarfone, E., Structural Relationships of the Unfixed Tectorial Membrane, Hear. Res., Vol.151 (2001), pp.41-47.

(25) Andoh, M. and Wada, H., Prediction of the Characteristics of Two Types of Pressure Waves in the Cochlea: Theoretical Considerations, J. Acoust. Soc. Am., Vol.116 (2004), pp.417-425.

(26) Tomotika, S. and Aoi, T., An Expansion Formula for the Drag on a Circular Cylinder Moving through a Viscous Fluid at Small Reynolds Numbers, Quart. J. Mech. Appl. Math., Vol.4 (1951), pp.401-406.

(27) Slepecky, N. and Chamberlain, S.C., Distribution and Polarity of Actin in the Sensory Hair Cells of the Chinchilla Cochlea, Cell Tissue Res., Vol.224 (1982), pp.15-24.

(28) Itoh, M. and Nakashima, T., Structure of the Hair Rootlets on Cochlear Sensory Cells by Tannic Acid Fixation, Acta Oto-Laryngol., Vol.90 (1980), pp.385390.

(29) Clark, J.A. and Pickles, J.O., The Effects of Moderate and Low Levels of Acoustic Overstimulation on Stereocilia and Their Tip Links in the Guinea Pig, Hear. Res., Vol.99 (1996), pp.119-128.

(30) Mountain, D.C. and Cody, A.R., Multiple Modes of Inner Hair Cell Stimulation, Hear. Res., Vol.132 (1999), pp.1-14. 